\title{
Management of posthaemorrhagic hydrocephalus with a subcutaneous ventricular catheter reservoir in premature infants
}

\author{
A LEONHARDT, ${ }^{*}$ H-H STEINER, $\dagger$ AND O LINDERKAMP* \\ Departments of *Paediatrics and + Neurosurgery, University of Heidelberg, Heidelberg, Federal Republic of \\ Germany
}

SUMMARY A subcutaneous ventricular catheter reservoir was inserted between the 16th and 28th (median 23rd) day of life in 13 premature infants (median birth weight $1220 \mathrm{~g}$, range 780-2110) for the treatment of posthaemorrhagic hydrocephalus. Aspiration of cerebrospinal fluid (median $6 \mathrm{ml}$, range 1-15) one to four (median two) times a day for 6 to 90 (median 40) days controlled the hydrocephalus as judged by clinical and ultrasonographic criteria. There were no serious complications, except skin breakdown in one patient. Intracranial pressure measured in five patients through the reservoir showed the efficacy of the treatment, as well as the unreliability of clinical and ultrasonographic criteria in determining the amount of cerebrospinal fluid that should be aspirated daily.

Management of posthaemorrhagic hydrocephalus in premature infants during the first weeks of life is difficult because of the low body weight and the associated serious medical problems in these severely compromised patients. Surgical methods of controlling the intracranial pressure include serial lumbar punctures, ${ }^{1-3}$ external ventricular drainage (usually through a subcutaneously tunnelled ${\text { catheter })^{2}}^{25}$ insertion of a ventriculoperitoneal shunt ${ }^{6}$ and placement of a subcutaneous ventricular catheter reservoir. ${ }^{78}$

Since 1982 we have used a ventricular catheter with subcutaneous reservoir for the treatment of posthaemorrhagic hydrocephalus. In five of our patients the intracranial pressure was measured through the reservoir. The aim of this retrospective study was to assess the efficacy and the complications of this particular treatment, and of measuring intracranial pressure through the reservoir.

\section{Patients and methods}

Between 1982 and 1987 a ventricular catheter with a subcutaneous reservoir (Holter-Hausner International) was placed in each of 13 premature infants with posthaemorrhagic hydrocephalus (table 1 ). The insertion of the reservoir was considered necessary if at least one of the criteria given in table 2 was met. The procedure took about 30 minutes under general anaesthesia and was done in the operating theatre. The ventricular catheter was positioned in the frontal horn of the right lateral ventricle, and the

Table 1 Characteristics of premature infants and courses of treatment of posthaemorrhagic hydrocephalus with subcutaneous reservoir $(n=13)$

\begin{tabular}{|c|c|c|}
\hline & Median & (range) \\
\hline Gestational age (weeks) & 29 & $(26-33)$ \\
\hline Birth weight $(\mathrm{g})$ & 1070 & $(740-2130)$ \\
\hline Haemorrhage grade $^{10}$ & 3 & $(2-4)$ \\
\hline Age at insertion of reservoir (days) & 23 & $(16-28)$ \\
\hline Weight at insertion of reservoir (g) & 1220 & $(780-2110)$ \\
\hline $\begin{array}{l}\text { Period during which aspiration of } \\
\text { cerebrospinal fluid carried out (days) }\end{array}$ & 40 & $(6-90)$ \\
\hline No of aspirations: & & \\
\hline No/day for each patient & $\begin{array}{r}2 \\
50\end{array}$ & $(1-4)$ \\
\hline \multicolumn{3}{|c|}{ Volume of cerebrospinal fluid aspirated (ml): } \\
\hline Volume at each aspiration & 6 & $(1-15)$ \\
\hline Total volume/each patient & 477 & $(76-1917)$ \\
\hline
\end{tabular}

Table 2 Criteria for insertion of a subcutaneous reservoir

- Increase in head circumference of more than $1 \mathrm{~cm} /$ week

- Progressive ventricular dilatation on ultrasound scans

- Obstructive posthaemorrhagic hydrocephalus (failure to control posthaemorrhagic hydrocephalus by lumbar punctures)

- Severe deterioration during lumbar puncture (for example, bradycardia, or apnoea) 
dome of the reservoir was positioned over the parietal bone. Prophylactic antibiotics were not given.

The reservoir was tapped with a small gauge ( 25 or 27) scalp vein needle, the operator wearing sterile gloves, and having carefully disinfected the skin. The aspirated volume of cerebrospinal fluid and the frequency of tapping were considered to be adequate if the following criteria were fulfilled: (i) increase in head circumference of less than 1 $\mathrm{cm} /$ week, (ii) unchanged or decreasing size of ventricles on ultrasound scans, (iii) normal tension of the fontanelle, and normal position of the cranial sutures. Occipitofrontal head circumference was measured and the fontanelle and the cranial sutures were palpated daily by the same person. Ultrasound scans of the brain with estimation of the ventricular size, as well as cell count, protein and glucose concentrations, and bacterial cultures of specimens of cerebrospinal fluid were carried out at least once a week. White blood cell count, and serum electrolyte and total serum protein concentrations were measured every other week.

In five patients a pressure transducer (Sensonor) was used to measure the intracranial pressure through the reservoir before and after aspiration of cerebrospinal fluid. An intracranial pressure of less than $6 \mathrm{~mm} \mathrm{Hg}$ was considered to be normal. ${ }^{9}$

For evaluation of the risk of the treatment the following possible complications were recorded: infection of the reservoir or sepsis (the presence of pathogens on culture of the cerebrospinal fluid), breakdown of the skin overlying the reservoir, intraventricular haemorrhage after aspiration of cerebrospinal fluid, leak from the reservoir, obstruction of the ventricular catheter, or low serum sodium $(<135 \mathrm{mmol} / \mathrm{l})$ or total protein $(<40 \mathrm{~g} / \mathrm{l})$ concentrations.

\section{Results}

The essential data concerning the treatment are shown in table 1 . Six patients weighed $1000 \mathrm{~g}$ or less at the time of insertion of the reservoir. Daily aspirations of the reservoir for more than $\mathbf{4 0}$ days were carried out in seven patients.

In all 13 patients, normal increase of occipitofrontal head circumference, unchanged or decreasing ventricular size, normal tension of the fontanelle, and normal position of cranial sutures were achieved. In 12 patients tapping was continued until a ventriculoatrial or ventriculoperitoneal shunt could be inserted with a low risk of complications. At the time of placement of the shunt, the median weight was $2320 \mathrm{~g}$ (range 1500-4500) and protein concentration in the cerebrospinal fluid was less than
$1.5 \mathrm{~g} / \mathrm{l}$. In one patient the posthaemorrhagic hydrocephalus resolved spontaneously and no further treatment was necessary. None of the patients died.

Intracranial pressure was measured through the reservoir before and after aspiration of cerebrospinal fluid in five patients on 47 occasions (table 3). Aspiration of cerebrospinal fluid caused a decrease in intracranial pressure of between 2 and 11 (median 3) $\mathrm{mm} \mathrm{Hg}$. In fourteen of the 47 measurements before, and in two of the 47 measurements after aspiration of cerebrospinal fluid, the intracranial pressure was raised (that is, above $6 \mathrm{~mm} \mathrm{Hg}$ ). In three patients intracranial pressure was raised and returned to normal or was unchanged within the normal range; in another patient, the raised intracranial pressure did not decrease during the treatment, and subsequently a ventriculoatrial shunt was inserted. The clinical course and the ventricular size on ultrasound scans corresponded with the degree of intracranial pressure in these four patients. In one patient, however, intracranial pressure was raised to $14 \mathrm{~mm} \mathrm{Hg}$, although clinical signs and ventricular size on ultrasound scans did not show any signs of increasing intracranial pressure. This patient also had a ventriculoatrial shunt inserted.

The following complications occurred: skin breakdown $(n=1)$ - the reservoir was exchanged and prophylactic antibiotics were started; hyponatraemia $(n=8)$, and hypoproteinaemia $(n=2)$. In one patient erythrocytes were found in the cerebrospinal fluid during the fourth and fifth week after the reservoir had been inserted, but new intraparenchymal or intraventricular haemorrhages were not detectable on ultrasound examination. Neither a leak from the reservoir nor obstruction of the ventricular catheter was seen, nor did any infection occur that could be associated with the reservoir.

\section{Discussion}

The results of this study show that posthaemorrhagic hydrocephalus can be successfully treated with a subcutaneous ventricular catheter reservoir. Our

Table 3 Intracranial pressure measured through a subcutaneous reservoir on 47 occasions in five premature infants with posthaemorrhagic hydrocephalus before and after aspiration of cerebrospinal fluid

\begin{tabular}{lll}
\hline & Median (range) \\
\hline $\begin{array}{l}\text { Age (days) } \\
\text { Intracranial pressure (mm Hg): }\end{array}$ & 26 & $(19-89)$ \\
$\quad$ Before aspiration & & \\
$\quad \begin{array}{l}\text { After aspiration } \\
\text { Volume of cerebrospinal fluid aspirated (ml) }\end{array}$ & 5 & $\begin{array}{l}(1-19) \\
(2-7)\end{array}$ \\
\hline
\end{tabular}


findings confirm those of previous studies ${ }^{7}$ that also showed decreasing ventricle size on computed tomograms of the brain after aspiration of cerebrospinal fluid through a subcutaneous reservoir.

A common problem in the treatment of posthaemorrhagic hydrocephalus with a subcutaneous reservoir is to know how often and how much to tap. In the present study the frequency and volume were determined from clinical signs and the size of the ventricles on ultrasound scans. Ultrasound estimation of ventricle size is, however, sometimes difficult in infants with posthaemorrhagic hydrocephalus, especially if porencephalic cysts or asymmetrical ventricular enlargement have developed. Moreover tension of the fontanelle and increasing head circumference are not always sensitive signs of raised intracranial pressure. ${ }^{11} 12$

We therefore repeatedly measured the intracranial pressure through the reservoir in five infants (table 3). Most measurements of intracranial pressure were within the normal range, and this shows the efficacy of the treatment that was guided by clinical and ultrasonographic criteria. Some of the high measurements of intracranial pressure were found at the beginning of the treatment and decreased subsequently. In general, clinical and ultrasonographic signs corresponded with the degree of the intracranial pressure. We did, however, find high intracranial pressure in one patient though clinical signs and ultrasound scans did not indicate any increase in intracranial pressure. The control and guidance of the treatment by only clinical and ultrasonographic criteria, therefore, is not sufficient in some patients, but may be improved by the measurement of intracranial pressure through the reservoir. Intermittent peaks of intracranial pressure between the taps cannot be ruled out even if intracranial pressure is normal at the time of aspiration of cerebrospinal fluid. Continuous monitoring of intracranial pressure could probably further increase the efficacy of the treatment until clinical and ultrasonographic criteria are stable.

We found skin breakdown in one patient four weeks after insertion of the reservoir. At this time the daily volume of cerebrospinal fluid being aspirated had been decreased in an attempt to wean the patient off the taps. Rising intracranial pressure with subsequent head growth had probably increased the vulnerability of the skin overlying the reservoir.

In one of our patients we found fresh erythrocytes in the cerebrospinal fluid during the fourth and fifth weeks after the insertion of the reservoir. On ultrasound scans neither new intraventricular nor intraparenchymal haemorrhages were detectable. Rupture of a small intracranial blood vessel cannot be ruled out, however. The intermittent aspiration of cerebrospinal fluid caused fluctuations in intracranial pressure of up to $11 \mathrm{~mm} \mathrm{Hg}$. Thus rupture of intracranial vessels may be the result of rapid aspiration of cerebrospinal fluid.

Hyponatraemia was found in eight infants, and hypoproteinaemia in two. The extent of hypoproteinaemia and hyponatraemia were not associated with the amount of cerebrospinal fluid that was being aspirated, but sodium and protein supplementation were not standardised in these infants. Sodium and protein depletion, which are theoretically possible if large amounts of cerebrospinal fluid are aspirated, can be avoided by giving sodium and protein supplements.

Obstruction of the ventricular catheter did not occur nor was it seen in another series of patients with posthaemorrhagic hydrocephalus treated with a ventricular catheter reservoir. ${ }^{8}$

Cerebrospinal fluid infections did not occur in spite of frequent tappings during a period of up to 90 days (table 1). Marlin ${ }^{7}$ reported two infections associated with insertion of the reservoir in a group of 12 patients with a maximum period of treatment of 101 days. In another study ${ }^{8}$ no infections of the reservoir were seen in 20 patients with a maximum period of treatment of 48 days. Thus the risk of infection associated with the reservoir is low. External drainage with a subcutaneously tunnelled distal catheter ${ }^{4} 5$ was also associated with a low risk of infection, but the period of treatment did not exceed 42 days. Infections associated with shunts occurred in $25 \%$ of infants with ventriculoperitoneal shunts. ${ }^{6}{ }^{1314}$ In infants weighing more than $2000 \mathrm{~g}$ early insertion of a ventriculoperitoneal shunt has a low risk of infection, ${ }^{6}$ but a high protein content or debris in the cerebrospinal fluid may cause obstruction of the ventricular catheter.

In most premature infants with posthaemorrhagic hydrocephalus, temporary measures for control of raised intracranial pressure are necessary until the insertion of an internal shunt can be carried out under optimal conditions after resolution of the patient's medical problems. Our data, together with other reports on the treatment of posthaemorrhagic hydrocephalus in small preterm infants, suggest the following approach: after the diagnosis of posthaemorrhagic hydrocephalus, serial lumbar punctures should be performed. If lumbar punctures do not succeed in controlling the intracranial pressure or if the infant deteriorates critically during the puncture, a subcutaneous ventricular catheter reservoir should be inserted if the baby weighs less than $2000 \mathrm{~g}$. The volume and frequency of tapping of the reservoir is determined by clinical signs (tense fontanelle and increasing head circumference) and ultrasound scans. Direct intracranial pressure 
measurements through the reservoir may increase the efficacy and the safety of the treatment. A ventriculoatrial or ventriculoperitoneal shunt should be inserted if resolution of the posthaemorrhagic hydrocephalus has not taken place by the time the infant weighs $2000 \mathrm{~g}$, and if the protein concentration of the cerebrospinal fluid is low.

\section{References}

${ }^{1}$ Papile LA, Burstein J, Burstein R, Koffler H, Koops BL. Posthemorrhagic hydrocephalus in low-birth-weight infants: treatment by serial lumbar punctures. $J$ Pediatr 1980;97:273-7.

2 Allan WC, Volpe JJ. Periventricular-intraventricular hemorrhage. Pediatr Clin North Am 1986;33:47-63.

3 Kreusser KL, Tarby TH, Kovnar EK, Taylor DA, Hill A, Volpe JJ. Serial lumbar punctures for at least temporary amelioration of neonatal posthemorrhagic hydrocephalus. Pediatrics 1985;75: 719-24.

${ }^{4}$ Harbaugh RE, Saunders RL, Edwards WH. External ventricular drainage for control of posthemorrhagic hydrocephalus in premature infants. J Neurosurg 1981;55:766-70.

5 Amato M, Guggisberg Ch, Kaiser G. Treatment of progressive posthemorrhagic hydrocephalus with temporary external ventricular drainage. Helv Paediatr Acta 1986;41:317-24.

6 James HE, Bejar R, Gluck L, et al. Ventriculoperitoneal shunts in high risk newborns weighing under 2000 grams: a clinical report. Neurosurgery 1984;15:198-202.

${ }^{7}$ Marlin AE. Protection of the cortical mantle in premature infants with posthemorrhagic hydrocephalus. Neurosurgery 1980;7:464-8.

${ }^{8}$ McComb JG, Ramos AD, Platzker ACG, Henderson DJ, Segall HD. Management of hydrocephalus secondary to intraventricular hemorrhage in the preterm infant with a subcutaneous ventricular catheter reservoir. Neurosurgery 1983;13:295-300.

9 Hill A. Intracranial pressure measurements in the newborn. Clin Perinatol 1985;12:161-78.

10 Papile LA, Burstein J, Burstein R, Koffler H. Incidence and evolution of subependymal and intraventricular hemorrhage: a study of infants with birth weights of less than 1,500 gm. Pediatrics 1978;92:629-34.

${ }^{11}$ Kaiser AM, Whitelaw AGL. Intracranial pressure estimation by palpation of the anterior fontanelle. Arch Dis Child 1987;62: 516-17.

12 Korobkin $\mathbf{R}$. The relationship between head circumference and the development of communicating hydrocephalus in infants following intraventricular hemorrhage. Pediatrics 1975;56:74-7.

13 Hahn YS, McLone DG, Raimondi AJ, Frisbe CL. Surgical outcome of preterm newborns with severe periventricularintraventricular hemorrhage and post-hemorrhagic hydrocephalus. American Society for Pediatric Neurosurgery: concepts in pediatric neurosurgery. Basle: Karger, 1983.

14 Scarff TB, Anderson DE, Anderson CL, Caldwell CC. Complications of ventriculoperitoneal shunts in premature infants. American Society for Pediatric Neurosurgery: concepts in paediatric neurosurgery. Basle: Karger, 1983.

Correspondence to: Dr A Leonhardt, Children's Hospital, Im Neuenheimer Feld 150, D-6900 Heidelberg, Federal Republic of Germany.

Accepted 21 August 1988

\section{Commentary}

\section{BANNISTER}

\section{Consultant Paediatric Neurosurgeon, Manchester}

The early management of hydrocephalus after intraventricular haemorrhage in low birthweight, premature infants of low gestational age continues to give problems. Those who care for these tiny infants are still seeking the ideal method of treatment that will control the hydrocephalus but neither produce infection of the cerebrospinal fluid or damage the central nervous system. By inserting a ventricular catheter attached to a reservoir situated beneath the scalp, the authors achieved satisfactory control in 13 infants with gestational ages ranging from 26 to 33 weeks, and with birth weights of between 740 and $2130 \mathrm{gm}$ by repeatedly tapping the reservoir, sometimes more than once a day, for periods lasting up to 90 days. While the necessity to carry out the taps and the volume of fluid removed was largely determined by the clinical signs and the size of the ventricles measured by ultrasound scanning, note was also taken of intracranial pressure measurements made through the reservoir before and after removal of the cerebrospinal fluid. The authors contend that measurement of the intracranial pressure probably increased the efficacy of their treatment.

In this small series satisfactory control of the hydrocephalus was achieved, but not without complications. Wound breakdown occurred in one case, and in another fresh blood was found in the cerebrospinal fluid after a tap. No infections or blockages of the ventricular catheters occurred, but it has to be stressed that this was an extremely small series. The method allows easy repeated removal of cerebrospinal fluid from the lateral ventricles and is a convenient way of measuring intracranial pressure, but it has the disadvantage that it requires an operation to be performed on a small and often frail premature infant, and insertion of a ventricular catheter does cause some local cortical damage. What the method does not permit is continuous removal of cerebrospinal fluid, and therefore-as the authors point out-the intracranial pressure is not smoothly controlled. Whether or not this is important for long term development of the brain has yet to be determined. Other methods of intermittent removal of cerebrospinal fluid from the ventricular system probably achieve results comparable to those reported by the authors.

Though the method described has some attractive features, in particular its use of intracranial pressure 